\title{
HDAC6 is a Regulator of CTL Function through Control of Lytic Granule Dynamics
}

Norman Nunez-Andrade ${ }^{1,2}$, Francisco Sanchez-Madrid ${ }^{1,2^{*} \#}$ and Noa Beatriz Martin-Cofreces ${ }^{1,2 \#}$

${ }^{1}$ Servicio de Inmunología, Hospital Universitario de la Princesa, UAM, IIS-IP, Madrid, Spain

${ }^{2}$ Area of Vascular Pathophysiology, Laboratory of Intercellular Communication, Fundación Centro Nacional de Investigaciones Cardiovasculares-Carlos III, Madrid, Spain

\#These two authors contributed equally

*Corresponding author: Francisco Sánchez-Madrid, Laboratorio de Comunicación Intercelular, Servicio de Inmunología-Planta, Instituto de Investigación Sanitaria Princesa, Hospital de La Princesa, Diego de León 62, 28006, Madrid, Spain, Tel: +34915202307; Fax: +34915202374; E-mail: fsmadrid@salud.madrid.org

Rec date: May 19, 2016; Acc date: Jun 21, 2016; Pub date: Jun 23, 2016

Copyright: (c) 2016 Núñez-Andrade N, et al. This is an open-access article distributed under the terms of the Creative Commons Attribution License, which permits unrestricted use, distribution, and reproduction in any medium, provided the original author and source are credited.

\begin{abstract}
Viral infections involve specific stress exposure that can influence the quality and average lifespan of an organism. The immune system acts through virus clearance from the organism. Many aspects of immune cells accounting for this response are still under study. Here, we review recent aspects of the molecular mechanisms involved in the delivery of the lethal hit by Cytotoxic T lymphocytes.
\end{abstract}

Keywords: HDAC6; Kinesin; Lytic granule; Microtubule; Centrosome

\section{Introduction}

The protection of the organism against viral infections is performed through the action of a specialized population of CD8 T cells, named the cytotoxic T cells (CTLs). CTLs clear virus-infected cells through different mechanisms, such as FAS/FAS-L pathway activation, the secretion of pro-inflammatory cytokines (e.g. IFN- $\gamma$ and TNF- $\alpha$ ), and the polarized exocytosis of lytic granules at the synaptic cleft formed with the target cell upon antigen recognition (Figures 1 and 2). The lethal hit mediated by the effector proteins contained in the lytic granules requires the establishment of specific cell contacts with targets $[1,2]$. The centrosome relocation to these cell contacts is an important step to help the polarized secretion of lytic granules through the action of microtubules-related motor proteins, which transport the granules across cytoplasm $[3,4]$. Our study demonstrates that HDAC6, a class IIb histone deacetylase that uses acetylated tubulin at Lys40 as substrate [5], exerts an important role in the transport and exocytosis of these granules in CTLs [6].

The study of different HDACs has been performed with paninhibitors, such as Trichostatin A (TSA) or Vorinostat (suberanilohydroxamic acid; SAHA), that favor the suppressor activity of regulatory $\mathrm{T}$ cells (Tregs) and can therefore be useful for autoimmune diseases treatment or GVHD (graft versus host disease) [7,8]. Indeed, the use of HDAC6-deficient mice has demonstrated its role on GVHD based on the modulation of Tregs, which is performed through its interaction with the Hsp90 chaperone [9]. Knock-out mouse for HDAC9 (a class IIa HDAC) also presents an increased activity of Tregs, therefore preventing autoimmune colitis [10]. The interaction of HDAC6 and HDAC9 has been recently demonstrated. It is dependent on the second catalytic domain of HDAC6 [11], which is thought to use $\alpha$-tubulin as substrate since its mutation lowers the catalytic rate of the enzyme 5000-fold [12]. In our study, HDAC6deficient CTLs show impaired cytotoxic activity in vitro and in vivo concomitant with the decreased secretion of their lytic content upon $\mathrm{T}$
Cell Receptor (TCR) activation. By contrast, neither the strength of TCR activation nor IFN- $\gamma$ secretions were affected [6]. To analyze TCR activation, we activated isolated CTLs from WT and Hdac6-/- mice with monoclonal anti-CD3 plus anti-CD28 antibodies and studied the phosphorylation of downstream signaling molecules PLC $\gamma 1$ and Erk1/2 at specific residues (Y783 and T202/Y204, respectively) at different times by western blot. We indeed measured the increase of global intracellular calcium flux in real time [6] through flow cytometry [13], which is needed for complete TCR activation and the secretion process [14]. We found no defects for these parameters in HDAC6-deficient CTLs, in agreement with the correct IFN $\gamma$ production and secretion. In contrast, the acetylation of tubulin at Lys40 was clearly increased in Hdac6-/- resting and activated CTLs [6], as described before [15].
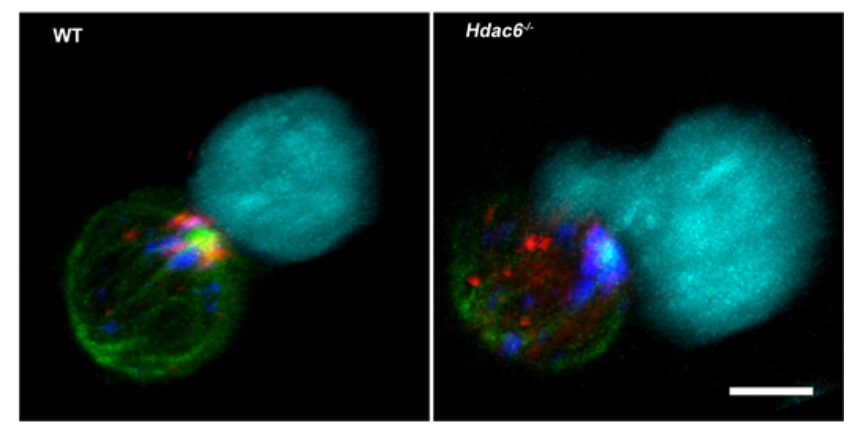

Figure 1: Localization of the centrosome and lytic granules in Hdac6-/- CTLs. Killing immune synapse formed by WT (left) or Hdac6-/- (right) cytotoxic T lymphocyte bearing a OVA-specific transgenic TCR (OT-I) and a target cell (Cyan; EL4 cell) presenting the specific OVA257-264 peptide (SIINFEKL), stained with antibodies against Cathepsin D (red) and CD107a (blue; LAMP1) for lytic granules, and a-tubulin for centrosome and microtubules (green). Bar, $5 \mu \mathrm{m}$. 
Citation: Nunez-Andrade N, Sanchez-Madrid F, Martin-Cofreces NB (2016) HDAC6 is a Regulator of CTL Function through Control of Lytic Granule Dynamics. Single Cell Biol 5: 143. doi:10.4172/2168-9431.1000143

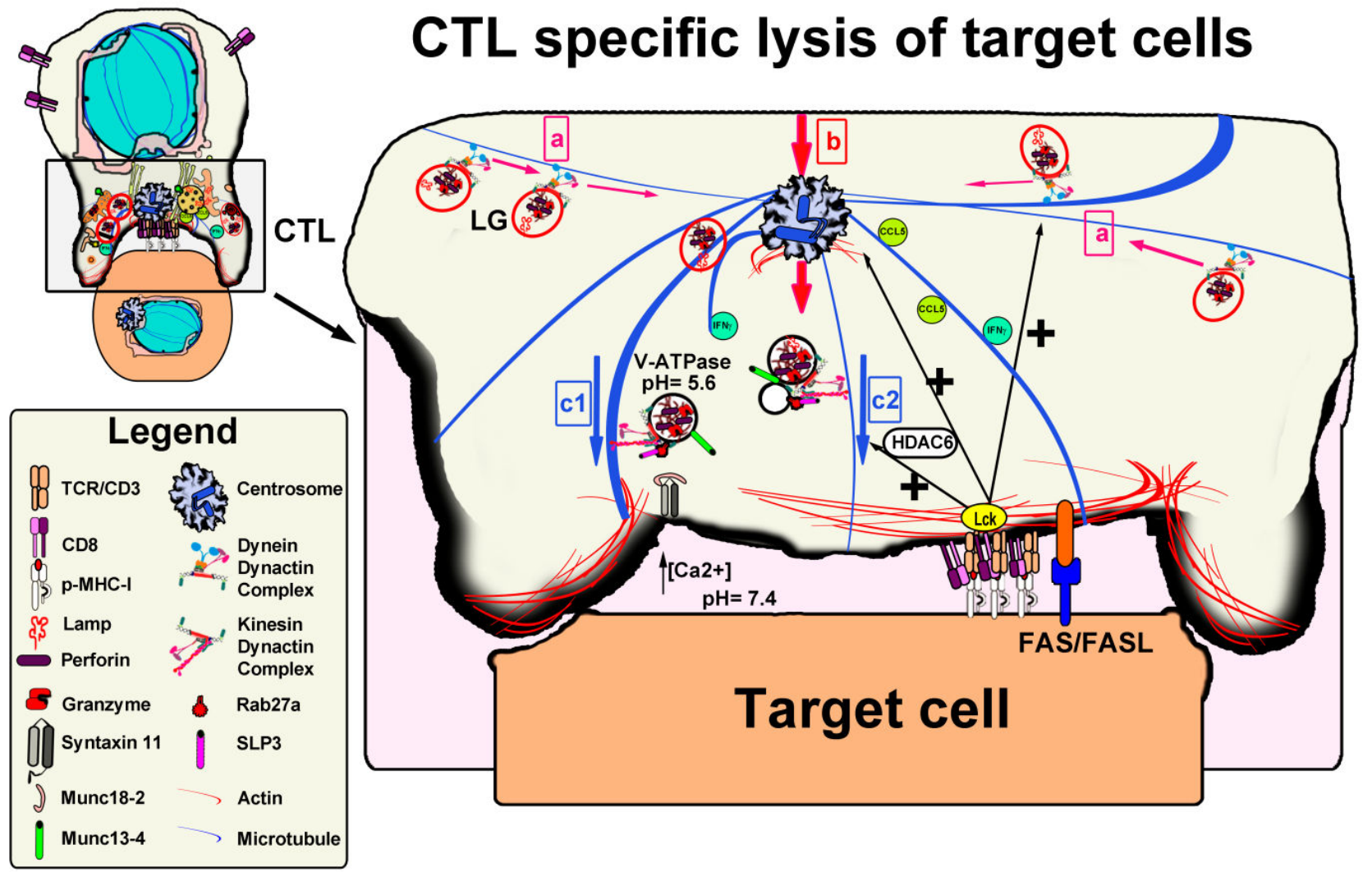

Figure 2: Proposed model for Cell Target killing. The formation of a lytic immune synapse is mediated by the specific peptide-MHC-I complex (p-MHC-I) recognition by the T Cell Receptor (TCR) and subsequent Lck activation. CTLs are not killed by their own lytic mediators due to the low $\mathrm{pH}$ present in the Lytic Granules (LG) owing to action of V-ATPase pump and aggregation (serglycine lattice). LG initially congregate at the centrosome (a) by minus-end-directed dynein molecular motor action. The centrosome polarizes (b) towards the target cell. Finally, LG move from the centrosome to the cell-cell contact area through the Kinesin-1-Rab27a-Slp3 complex (c) to secrete their content. This can be performed by LG that include all the mediators needed for docking at the plasma membrane, priming and secretion at the synaptic cleft (c1), or by exocytic vesicles that bear fusion mediators that collaborate with LG (c2). Dynactin complex can support Kinesin-1. HDAC6 regulates the formation of these complexes and favors centrosome polarization. FAS/FASL interaction can also mediate cell target killing.

The activity of HDAC6 in CD4+ T cells was previously analyzed in our laboratory after TCR activation; we found a more prominent effect on the correct $\mathrm{T}$ cell activation upon its over-expression than with its inactivation by genetic or chemical means. At short times upon TCR triggering, between 1-2 minutes, there is an effective deacetylation of a-tubulin at Lys40, which is in contrast largely acetylated at longer times [16]. Since acetylation of microtubules is considered a mark of stabilization in mammalian cells [17], we considered this initial HDAC6-dependent $\alpha$-tubulin deacetylation [16] a hallmark of the well-known reorganization of the microtubule network in CTLs upon target cell recognition with active centrosomal polarization at the cellcell contact [18]. The increase in acetylation at longer times of activation $[6,16]$ is most likely a requirement for the stabilization of the microtubular network that is newly polymerized at the immune synapse in formation. The growing of microtubules at the IS is needed for transport and full $\mathrm{T}$ cell activation $[19,20]$. In our present study in CD8+ CTLs, the increase in a-tubulin acetylation is effectively observed upon 5-20 minutes after TCR activation in WT cells. Loss of HDAC6 and hyper-acetylation of $\alpha$-tubulin does not seem to prevent TCR activation in CTLs and the centrosome polarization towards the target cell is even improved in these cells, correlating to the increased a-tubulin acetylation detected [6]. This is in agreement with an inhibition of centrosome polarization to the IS upon HDAC6 overexpression in CD4+ T cells, that is recovered by TSA treatment [16]. In contrast, a recent study with a new deacetylase inhibitor, ACY-1215, which shows a 10-fold more selectivity against HDAC6 than HDAC1/2/3 and slight activity against HDAC8, does find that CD8+ T cells do not activate correctly their TCR-dependent pathways and 
attributes this effect to HDAC6 interaction with Hsp90 [21]. Hsp90 is important for regulation of Lck conformation and activity [22], a major kinase for the initiation of TCR-downstream signaling, and helps the maintenance of a pre-activated pool of Lck in T cells [23]. It is therefore conceivable that the observed effects on TCR activation by this inhibitor may rely on other HDACs, such as in HDAC1 inhibition, which has been shown to prevent CD8+ $\mathrm{T}$ cell homeostasis and antiviral response against LCMV [24]. Indeed, TSA treatment in CD4+ $\mathrm{T}$ cells prevents the interaction of ZAP70 and phosphorylated-CD3 $\zeta$ with $\alpha$-tubulin, probably affecting final $\mathrm{T}$ cell activation. In addition, over-expression of HDAC6 completely prevented IL-2 production by SEE-activated CD4+ T cells (Staphylococcus aureus Enterotoxin E). This inhibition could not be relieved by PMA and ionophore treatment, pointing to a defect in intracellular traffic rather than a signaling one [16]. Remarkably, adoptive transfer of CD8+ T cells into Rag-/- recipients demonstrated that the presence of HDAC6 is relevant for protection against Vaccinia infection. Hence, HDAC6-deficient cells were less efficient in the clearance of infected cells, but not in recognizing them or in their ability to proliferate. Rather, HDAC6deficient cells showed an increased ability to proliferate at longer times [6].

The movement of the lytic granules towards the target cell depends on microtubules. CTLs are not killed by their own lytic mediators, which is attributed to the low $\mathrm{pH}$ present in the lytic granules thanks to the V-ATPase pump action and their aggregation with a serglycine lattice [25]. Upon the interaction of the TCR with a specific peptideMHCI complex, different organelles reorganize at the cytosol; in a first step, lytic granules congregate at the centrosome through dynein-based transport along microtubules [4]. Afterwards, the centrosome polarizes towards the target cell bringing the Golgi apparatus and lytic granules together, which facilitates polarized secretion [26]. Finally, the lytic granules move from the centrosome towards the cell-cell contact area through the action of a complex formed by Kinesin-1-rab27a-slp3 [27]. Defects in Rab27a underlies the Griscelli syndrome type, which causes a partial albinism due to defects in melanosomes transport and an immunodeficiency partly due to inadequate cytotoxic killing activity, related to its interaction with myosin Va (Griscelli Syndrome type 1; lack of transfer of vesicles from microtubules to actin filaments) [3]. Rab27a is needed for LG secretion [28]. Dynactin complex can also cooperate with kinesin in the movement of lytic granules. Our finding that the interaction of HDAC6 with kinesin-1 is detected upon TCR activation points to a role for this plus-end directed motor complex to favor and stabilize the dynamics of lytic granules at their terminal transport to effectively kill the target cell. HDAC6 coimmunoprecipitates with KLC1 (kinesin light chain 1) in WT CTLs upon TCR activation and prevents correct conformation of the dynactin complex in HDAC6-deficient CTLs [6]. KLC1 forms the kinesin-1 complex together with KIF5B (kinesin heavy chain) in CTLs [27].

The secretion of lytic granules and cytokines seems to follow different routes at the killing immune synapses respect to the cytokinebearing vesicles. Lytic granule secretion involves an additional step that requires the fusion of exocytic vesicles coming from recycling and late endosomes and provides the lytic granules with the essential docking and priming machinery to deliver their content at the synaptic cleft, such as Syntaxin 1, Munc 13-, Munc18-2 [3,29]. Defects in these proteins, as well as in perforin cause different FHL syndromes (Familial Hemophagocytic Lymphohistiocytosis); autosomal and often lethal recessive disorders with occurrence of HLH (Hemophagocytic lymphohistiocytic syndrome). We detected higher proliferation of
CD8+ T cells at late stages of infection and normal secretion of IFN $\gamma$ with low target cell killing, all of them hallmarks of the disease, although we did not observe the development of an HLH-like phenotype in HDAC6-deficient mouse upon infection with Vaccinia virus [6]. It is therefore conceivable that a chronic infection, such as the one provoked by LCMV, rather than an acute one is required to provoke the appearance of this syndrome. Novel insights into the function of different HDACs, such as HDAC8, which blockade disrupts the centrosome and precludes correct endolysosomal biogenesis [30], HDAC9 or HDAC1 may help to elucidate the role of HDAC6 in the cytotoxic process and to use different chemical inhibitors as tools to target CTL functions.

\section{Acknowledgments}

This study was supported by grants SAF2014-55579-R from the Spanish Ministry of Economy and Competitiveness, INDISNETS2011/BMD-2332 from the Comunidad de Madrid (Fondos FEDER), ERC-2011-AdG 294340-GENTRIS from EU. Red Cardiovascular RD 12-0042-0056 from Instituto Salud Carlos III (ISCIII). The Centro Nacional de Investigaciones Cardiovasculares (CNIC, Spain) is supported by the Spanish Ministry of Science and Innovation and the Pro-CNIC Foundation.

\section{Competing Interest}

Authors declare no competing interests.

\section{References}

1. Pardo J, Aguilo JI, Anel A, Martin P, Joeckel L, et al. (2009) The biology of cytotoxic cell granule exocytosis pathway: granzymes have evolved to induce cell death and inflammation. Microbes Infect 11: 452-459.

2. Stinchcombe JC, Griffiths GM (2007) Secretory mechanisms in cellmediated cytotoxicity. Annu Rev Cell Dev Biol 23: 495-517.

3. de Saint Basile G, Ménasché G, Fischer A (2010) Molecular mechanisms of biogenesis and exocytosis of cytotoxic granules. Nat Rev Immunol 10: 568-579.

4. Stinchcombe JC, Majorovits E, Bossi G, Fuller S, Griffiths GM (2006) Centrosome polarization delivers secretory granules to the immunological synapse. Nature 443: 462-465.

5. Hubbert C, Guardiola A, Shao R, Kawaguchi Y, Ito A, et al. (2002) HDAC6 is a microtubule-associated deacetylase. Nature 417: 455-458.

6. Núñez-Andrade N, Iborra S, Trullo A, Moreno-Gonzalo O, Calvo E, et al. (2016) HDAC6 regulates the dynamics of lytic granules in cytotoxic T lymphocytes. J Cell Sci 129: 1305-1311.

7. Akimova T, Ge G, Golovina T, Mikheeva T, Wang L, et al. (2010) Histone/ protein deacetylase inhibitors increase suppressive functions of human FOXP3+ Tregs. Clin Immunol 136: 348-363.

8. Tao R, de Zoeten EF, Ozkaynak E, Chen C, Wang L, et al. (2007) Deacetylase inhibition promotes the generation and function of regulatory T cells. Nat Med 13: 1299-1307.

9. de Zoeten EF, Wang L, Butler K, Beier UH, Akimova T, et al. (2011) Histone deacetylase 6 and heat shock protein 90 control the functions of Foxp3(+) T-regulatory cells. Mol Cell Biol 31: 2066-2078.

10. de Zoeten EF, Wang L, Sai H, Dillmann WH, Hancock WW (2010) Inhibition of HDAC9 increases T regulatory cell function and prevents colitis in mice. Gastroenterology 138: 583-594.

11. Salian-Mehta S, Xu M, McKinsey TA, Tobet S, Wierman ME (2015) Novel Interaction of Class IIb Histone Deacetylase 6 (HDAC6) with Class IIa HDAC9 Controls Gonadotropin Releasing Hormone (GnRH) Neuronal Cell Survival and Movement. J Biol Chem 290: 14045-56. 
Citation: Nunez-Andrade N, Sanchez-Madrid F, Martin-Cofreces NB (2016) HDAC6 is a Regulator of CTL Function through Control of Lytic Granule Dynamics. Single Cell Biol 5: 143. doi:10.4172/2168-9431.1000143

Page 4 of 4

12. Zou H, Wu Y, Navre M, Sang BC (2006) Characterization of the two catalytic domains in histone deacetylase 6. Biochem Biophys Res Commun 341: 45-50.

13. June $\mathrm{CH}$, Moore JS (2004) Measurement of intracellular ions by flow cytometry. Curr Protoc Immunol Chapter 5: Unit 5.

14. Premack BA, Gardner P (1992) Signal transduction by T-cell receptors: mobilization of $\mathrm{Ca}$ and regulation of $\mathrm{Ca}$-dependent effector molecules. Am J Physiol 263: C1119-1140.

15. Gao YS, Hubbert CC, Lu J, Lee YS, Lee JY, et al. (2007) Histone deacetylase 6 regulates growth factor-induced actin remodeling and endocytosis. Mol Cell Biol 27: 8637-8647.

16. Serrador JM, Cabrero JR, Sancho D, Mittelbrunn M, Urzainqui A, et al. (2004) HDAC6 deacetylase activity links the tubulin cytoskeleton with immune synapse organization. Immunity 20: 417-428.

17. Piperno G, LeDizet M, Chang XJ (1987) Microtubules containing acetylated alpha-tubulin in mammalian cells in culture. J Cell Biol 104 289-302.

18. Geiger B, Rosen D, Berke G (1982) Spatial relationships of microtubuleorganizing centers and the contact area of cytotoxic T lymphocytes and target cells. J Cell Biol 95: 137-143.

19. Martín-Cófreces NB, Baixauli F, López MJ, Gil D, Monjas A, et al. (2012) End-binding protein 1 controls signal propagation from the $\mathrm{T}$ cell receptor. EMBO J 31: 4140-4152.

20. Blas-Rus N, Bustos-Moran E, Perez de Castro I, de Carcer G, Borroto A et al. (2016) Aurora A drives early signalling and vesicle dynamics during T-cell activation. Nat Commun 7: 11389.

21. Tsuji G, Okiyama N, Villarroel VA, Katz SI (2015) Histone deacetylase 6 inhibition impairs effector CD8 T-cell functions during skin inflammation. J Allergy Clin Immunol 135: 1228-1239.
22. Giannini A, Bijlmakers MJ (2004) Regulation of the Src family kinase Lck by Hsp90 and ubiquitination. Mol Cell Biol 24: 5667-5676.

23. Nika K, Soldani C, Salek M, Paster W, Gray A, et al. (2010) Constitutively active Lck kinase in $\mathrm{T}$ cells drives antigen receptor signal transduction. Immunity 32: 766-777.

24. Tschismarov R, Firner S, Gil-Cruz C, Göschl L, Boucheron N, et al (2014) HDAC1 controls CD8+ T cell homeostasis and antiviral response. PLoS One 9: e110576.

25. Clark R, Griffiths GM (2003) Lytic granules, secretory lysosomes and disease. Curr Opin Immunol 15: 516-521.

26. Bustos-Moran E, Blas-Rus N, Martín-Cófreces NB, Sánchez-Madrid F (2016) Orchestrating lymphocyte polarity in cognate immune cell-cell interactions. Int Rev Cell Mol Biol In Press.

27. Kurowska M, Goudin N, Nehme NT, Court M, Garin J, et al. (2012) Terminal transport of lytic granules to the immune synapse is mediated by the kinesin-1/Slp3/Rab27a complex. Blood 119: 3879-3889.

28. Stinchcombe JC, Barral DC, Mules EH, Booth S, Hume AN, et al. (2001) $\mathrm{Rab} 27 \mathrm{a}$ is required for regulated secretion in cytotoxic $\mathrm{T}$ lymphocytes. J Cell Biol 152: 825-834.

29. Pachlopnik Schmid J, Côte M, Ménager MM, Burgess A, Nehme N, et al. (2010) Inherited defects in lymphocyte cytotoxic activity. Immunol Rev 235: 10-23.

30. Yamauchi Y, Boukari H, Banerjee I, Sbalzarini IF, Horvath P, et al. (2011) Histone deacetylase 8 is required for centrosome cohesion and influenza A virus entry. PLoS Pathog 7: e1002316. 